\title{
Histopathological Study of Gallbladder Carcinoma and its Mimics with Role of Carcinoembryonic Antigen Immunomarker in Resolving Diagnostic Difficulties
}

\begin{abstract}
Background: Gallbladder carcinoma (GBC) sometimes presents with nonspecific signs, without forming a mass, mimicking benign gallbladder (GB) diseases. On the contrary, benign GB diseases may mimic GBC. Material and Methods: We retrospectively reviewed 107 cases over a period of 3 years (May 2012-April 2015), which included 41 review cases and 66 departmental cases. Carcinoembryonic antigen (CEA) immunomarker expression was done. Results: In 27 of the 41 review cases, the diagnoses were benign diseases of GB associated with mild-to-moderate dysplasia of mucosal glands; however, after review in our department, it was found that of these 27 cases, nine cases were actually well-differentiated adenocarcinoma of GB with diffuse CEA expression and were mis diagnosed as benign diseases of GB with dysplasia. In 32 out of 66 departmental cases, initial histopathological diagnoses were benign diseases of GB associated with dysplastic mucosal glands. After CEA staining, 11 out of these 32 cases turned out to be adenocarcinoma of the GB. Among the rest $34(34 / 66)$ departmental primary GBC cases, no CEA expression was seen in six cases, focal expression was seen in 12 cases, and diffuse expression was seen in 16 cases. No diffuse CEA expression was seen in benign diseases of the GB with dysplasia. Conclusion: GBC sometimes may not be diagnosed radiologically and grossly as it often presents without any mass and specific signs, which lead to under diagnosis. Some benign cases may mimic GBC and may complicate histological diagnosis. CEA expression may aid as an additional diagnostic aid in resolving diagnostic dilemmas.
\end{abstract}

Keywords: Carcinoembryonic antigen, gallbladder carcinoma, misdiagnosis

\section{Introduction}

Cancer is one of the biggest medical problems the human has ever encountered. One of the important frontiers of carcinoma is the gastrointestinal tract malignancy, of which gallbladder carcinoma (GBC) is of special mention. GBC is the fifth most common malignancy of the alimentary tract. ${ }^{[1]}$ Its incidence shows geographical and ethnic variations. High rates of GBC are seen in South Asian countries such as in India, Pakistan, Japan, and Korea. ${ }^{[2]}$ In India, GBC is the most common gastrointestinal cancer in women in North and Central India. ${ }^{[3,4]}$

There is a marked female predominance with female-to-male ratio being 3-4:1. ${ }^{[5]}$ Most patients diagnosed with GBC are in the sixth or seventh decade of life. ${ }^{[6]}$ Important risk factors include genetic backgrounds, gall stones, and abnormal choledochopancreatic junctions. ${ }^{[6]}$

\footnotetext{
This is an open access article distributed under the terms of the Creative Commons Attribution-NonCommercial-ShareAlike 3.0 License, which allows others to remix, tweak, and build upon the work non-commercially, as long as the author is credited and the new creations are licensed under the identical terms.
}

For reprints contact: reprints@medknow.com
Symptoms of GBC often mimic those of chronic cholecystitis such as pain in the upper quadrant of abdomen, nausea, vomiting, or features of obstructive jaundice. Most of the GBCs arise in the fundus of the gall bladder (GB). Although some of the cases presented with mass, in majority of the cases, no mass can be detected either radiologically or on gross examination. ${ }^{[7]}$ They present with thickening of GB wall or mucosal ulceration, leading to underdiagnoses; such patients are eventually diagnosed later and have a poor prognosis. ${ }^{[8,9]}$ Further, some benign diseases of GB such as Rokitansky-Aschoff sinuses (RA sinuses) may mimic well-differentiated GBC.

Tumor markers can be used to aid histopathological diagnosis of GBC and its mimics. ${ }^{[10]}$

In this study, we highlight histopathological diagnostic dilemmas of GBC and its mimics

How to cite this article: Mondal SK, Bhattacharjee D, Mandal PK, Biswas S. Histopathological study of gallbladder carcinoma and its mimics with role of carcinoembryonic antigen immunomarker in resolving diagnostic difficulties. Indian J Med Paediatr Oncol 2017;38:411-5.

\section{Santosh Kumar \\ Mondal, Debashish Bhattacharjee, Palash Kumar Mandal', Saumitra Biswas}

Department of Pathology, Bankura Sammilani Medical College, Bankura, ' Department of Pathology, College of Medicine and Sagar Dutta Hospital, Kolkata, West Bengal, India
Address for correspondence: Dr. Santosh Kumar Mondal, Subarnabhumi Complex, Kamini III, Flat A302, 36 Gorakshabashi Road, Dumdum, Kolkata - 700 028, West Bengal, India.

E-mail: dr_santoshkumar@ hotmail.com

Access this article online

Website: www.ijmpo.org

DOI: 10.4103/ijmpo.jjmpo_230_15 Quick Response Code:

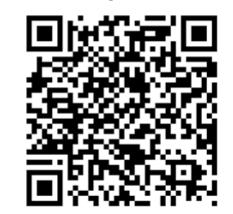


with application of carcinoembryonic antigen (CEA) in aiding histopathological diagnosis.

\section{Materials and Methods}

We retrospectively reviewed 107 cases over a period of 3 years (May 2012-April 2015), which include the data retrieved from histopathology files of our department. Patient particulars, detailed clinical histories, and investigation histories were collected from the Department of Surgery.

Of total 107 cases, 41 cases came for review at our institution and remaining 66 cases were departmental (which came to the Department of Pathology for histopathological examination from surgery operation theater). Of 41 review cases, 27 (27/41) had an initial diagnosis of benign GB diseases with dysplasia and remaining 14 (14/41) cases had an initial diagnosis of GBC. In case of 66 departmental cases, $32(32 / 66)$ cases had an initial diagnosis of benign GB diseases with dysplasia while remaining 34 cases (34/66) had an initial diagnosis of $\mathrm{GB}$ adenocarcinoma (GBC).

\section{Inclusion criteria}

a. Cases of departmental GBC which presented with mass, radiologically, grossly, and subsequently confirmed by histology

b. Cases of departmental GBC presented without mass on radiological investigations (ultrasonography or computed tomography scan) and few of which were clinically misdiagnosed as benign GB diseases (acute or chronic cholecystitis) but later diagnosed histologically as GBC

c. Cases of GB diseases (41/107) which came to our institution for review

d. Departmental cases histologically diagnosed as RA sinuses and adenomyomatosis of the GB but have some glands with dysplastic changes and were advised to rule out possibility of malignancy by clinical, radiological, and immunohistochemical (IHC) means.

\section{Exclusion criteria}

a. Cases of common benign GB disease with no histological diagnostic dilemmas such as acute or chronic cholecystitis

b. Cases of metastatic adenocarcinoma of the GB

c. Cases in which proper patient history, investigations record, or tissue blocks could not be retrieved.

CEA expression study by IHC was done in all 41 review cases and in all 66 of the departmental cases.

Paraffin blocks made from specimen were collected and the sections were used for hematoxylin and eosin staining and for IHC examinations. Monoclonal primary antibody for CEA was used for IHC. Standard procedures of IHC were followed.
For positive control, a section of colonic carcinoma known to be strongly positive for CEA was included.

Pattern of CEA expression was studied and recorded as apical, focal, or diffuse.

\section{Results}

Among 107 cases, 84 were females and 23 were males. Patients were in the age group of 50-70 years, $41(41 / 107)$ were review cases, and $66(66 / 107)$ were departmental cases.

In 27 of the review cases (27/41), the outside diagnoses were benign diseases of GB associated with dysplasia which included cases of RA sinuses associated with dysplasia and cases of adenomyomatosis of the GB associated with dysplasia. In 14 review cases (14/41), outside diagnoses were adenocarcinoma of the GB. In these 27 cases, after review in our department by thorough histopathological examination and CEA immunostaining, it was found that nine cases of well-differentiated adenocarcinoma of the GB with focal and diffuse CEA expression was mis diagnosed as benign diseases of GB with mild-to-moderate dysplasia by outside laboratories (cases with serial numbers 2, 4, 5,9, 13, 17, 18, 21, 22). Focal CEA expression was seen in three cases (cases 4, 9, 18) and diffuse expression was seen in rest six cases. In other review cases, outside diagnoses of benign diseases of GB associated with mild-to-moderate dysplasia was consistent with our diagnosis after review (both microscopically and immunohistochemically). In 14 other review cases (14/41), outside diagnoses of adenocarcinoma of the GB were also consistent with our diagnoses (both microscopically and immunohistochemically).

In 32 out of 66 departmental cases (32/66), initial histopathological diagnoses were either RA sinuses or adenomyomatosis of GB with mild-to-moderate dysplastic mucosal glands. After CEA staining, 11 cases (cases serial number 43, 49, 54, 58, 61, 67, 74, 78, 88, 99, 105) out of 32 cases turned out to be adenocarcinoma of GB with focal and diffuse CEA staining patterns. Focal expression was seen in four cases and diffuse expression in rest seven cases. Among 34 departmental primary GBC cases (34/66), 21 cases had no GB mass on gross examination and were diagnosed after histopathological examination. Rest 13 cases presented with GB mass radiologically and on gross examinations.

CEA staining in 34 departmental primary GBC cases showed no expression in 6 cases (6/34), focal expression was seen in 12 cases (12/34), and diffuse expression was seen in 16 cases (16/34). Hence, out of total 107 (41 review and 66 departmental) cases studied for IHC, there were $68 \mathrm{GBC}$ cases (34 departmental GBC cases and 14 review $\mathrm{GBC}$ cases in which initial review and our final diagnoses were GBC; 9 review cases and 11 departmental cases in which diagnoses were changed from benign GB diseases to GBC). 
CEA immunostaining was seen in 62 GBC cases $(91 \%)$. We noted no expression in six cases $(6 / 68)$, focal staining pattern was seen in 29 cases $(29 / 68)$, i.e., $42 \%$ of GBC cases and diffuse staining pattern was seen in 33 cases $(33 / 68,48 \%)$ of cases [Table 1].

Total number of cases with final diagnoses benign diseases of GB with dysplasia was 39 cases (18 review and 21 departmental cases). CEA expression was seen in 12 cases (12/39), i.e., $30 \%$ of cases.

CEA expression was apical in all 12 above cases.

\section{Discussion}

Most GBCs have no distinctive presenting features but often have symptoms resembling those of chronic cholecystitis. ${ }^{[11]}$ Laboratory findings in GBC are also not diagnostic and may include hyperbilirubinemia, elevated serum CEA, or CA 19-9. ${ }^{[12]}$ On radiological investigations, gall stones are found in about $80 \%$ of patients with GBC. ${ }^{[12,13]}$ Histopathological findings of GBC sometimes create diagnostic dilemmas; as sometimes, GBC may mimic benign GB diseases such as RA sinus, adenomyomatosis, and vice versa. ${ }^{[14]}$ Diagnosis of GBC has some potential pitfalls, which includes under diagnosis of invasive well-differentiated adenocarcinoma as deeply invasive RA sinuses, making an over diagnosis of GBC when benign conditions such as RA sinuses or adenomyomatosis of GB are present. CEA may aid in difficult histopathological diagnoses of GB diseases. CEA describes a set of highly related glycoproteins involved in cell adhesions. CEA is normally produced in gastrointestinal tissues during fetal development, but production stops before birth. CEA is an established marker of colorectal carcinoma, but there are few studies regarding expression patterns of CEA in GBC . To the best of our knowledge, there is only one such study in India. ${ }^{[15]}$

In our study, out of total 68 GBC cases, CEA immunostaining was seen in 62 GBC cases (91\%). We noted no expression in 6 cases $(6 / 68)$, focal staining pattern was seen in 29 cases (29/68), i.e., 42\% of GBC cases [Figure 1a], and diffuse staining pattern was seen in 33 cases $(33 / 68)$, i.e., $48 \%$ cases [Figure $1 \mathrm{~b}$ ]. In total 39 cases of benign diseases of GB with mild-to-moderate dysplasia, we found CEA expression in 12 cases (12/39), i.e., $30 \%$ of cases. CEA expression was apical in all 12 above cases. [Figure 1c]. Agrawal et al. reported that CEA immunostaining was observed in 42 of $51 \mathrm{GBC}$ cases $(82 \%)$.

They found that CEA staining pattern was apical in $13 / 51$ cases $(25 \%)$, focal pattern in $15 / 51$ cases $(29 \%)$ to diffuse in $14 / 51$ cases $(27 \%)$. They also found CEA expression in 18 of 68 chronic cholecystitis cases $(27 \%)$ and apical expression in all 18 cases along with focal cytoplasmic in nine cases. They concluded that diffuse cytoplasmic staining was found only in GBC. ${ }^{[15]}$

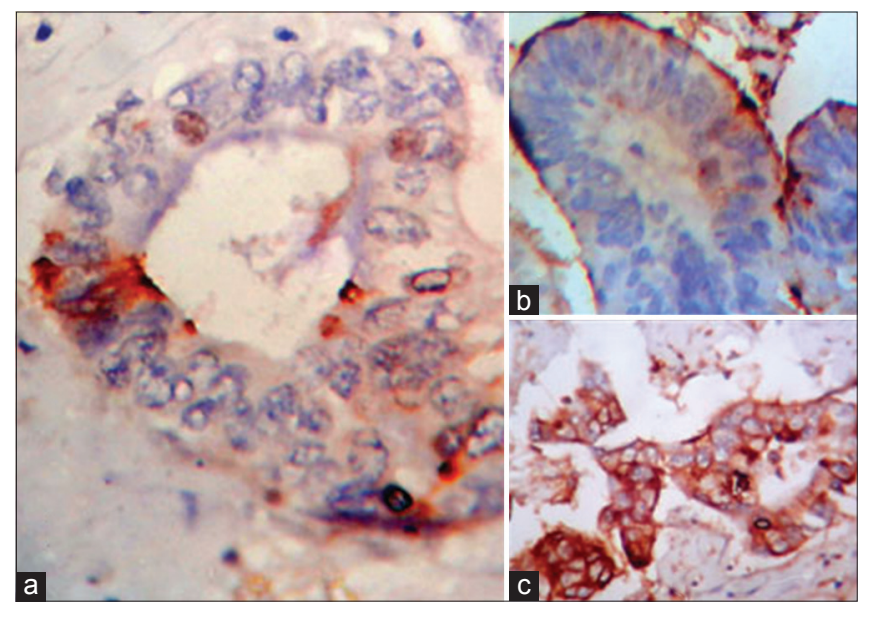

Figure 1: (a) Photomicrograph showing focal carcinoembryonic antigen expression in glands of gallbladder adenocarcinoma $(\times 400)$. (b) Photomicrograph showing apical carcinoembryonic antigen expression in lining of Rokitansky-Aschoff sinus involved by moderate dysplasia $(\times 400)$. (c) Photomicrograph showing diffuse Carcinoembryonic antigen expression in glands of gallbladder adenocarcinoma $(\times 400)$

In our study, we did not observe apical staining pattern in GBC cases; however, we also concluded that focal and diffuse CEA staining pattern was suggestive of GBC, and in the cases of benign GB diseases with dysplasia, either no CEA expression or apical CEA expression pattern is seen.

Roa et al. in their study on CEA expression in GB cancers observed that all cases of GBC were strongly positive for CEA, similar to our study, and out of 26 noncancerous GB diseases, in majority of the cases which expressed CEA, only weak CEA expression could be found. ${ }^{[16]}$

In another study by authors, Roa et al. observed that CEA was expressed in $89 \%$ cases of $\mathrm{GBC}$ of total $103 \mathrm{GBC}$ and only $12 \%$ cases of nontumoral lesions expressed CEA. In their study, they observed that CEA expression in nontumoral lesions was expressed only in apical parts of the cells. ${ }^{[17]}$

Albores-Saavedra et al. in their study on expression of CEA in GB epithelium concluded that from cases of dysplasia to invasive carcinoma, the amount of immunoreactive CEA progressively increases and shows a change in distribution. In malignant tumors, in addition to a stronger staining reaction, CEA could also been within the cytoplasm and even in gland secretions. ${ }^{[18]}$

In adenomyomatosis, there is thickening of the GB in a diffuse pattern while localized thickness of the GB is seen in adenomyoma. ${ }^{[19]}$ The GB epithelium extends downward as mucosal invaginations into the hyperplastic smooth muscle bundles and forms gland-like spaces or branched ducts [Figure $2 \mathrm{~d}$ and e].

These mucosal invaginations are lined by a single layer of tall columnar epithelial cells. These spaces contain bile, calculi, or mucus. Perineural invasion can also occur, but it does not indicate malignancy. ${ }^{[20]}$ 
Therefore, adenomyomatosis presenting with thickened GB wall, mucosal invaginations to smooth muscle bundles and sometimes with perineural invasion may mimic GB adenocarcinoma.

In few of our cases, misdiagnoses of adenomyomatosis with moderate dysplasia was made in histopathological examination, but after CEA staining (diffuse pattern), a diagnosis of well-differentiated adenocarcinoma of the GB was established.

Albores-Saavedra et al. reported nine cases of adenomyomatosis of GB with perineural invasion mimicking adenocarcinoma of the GB. ${ }^{[20]}$ Thus, in our case, adenocarcinoma of GB mimicked adenomyomatosis of GB with dysplasia while Albores-Saavedra et al. reported adenomyomatosis of GB mimicking GB adenocarcinoma.

In another cases, outside report was RA sinus with mild-to-moderate dysplasia. On CEA immunostaining, diffusely positive pattern was observed. After giving deeper section, histopathological examination was repeated and well-differentiated adenocarcinoma extending into the RA sinus was found [Figure $2 \mathrm{a}$ and $\mathrm{b}$ ]. There was the presence

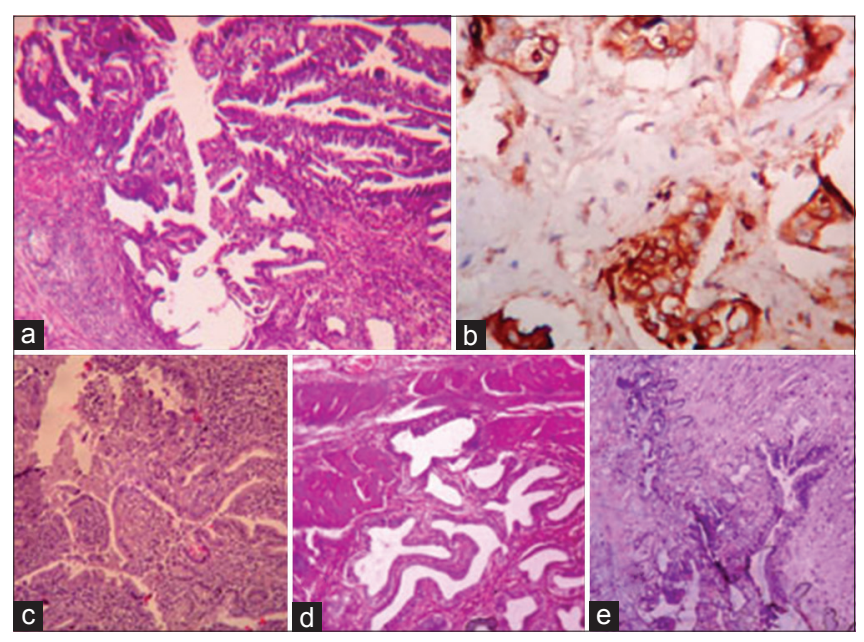

Figure 2: (a) Well differentiated adenocarcinoma along a Rokitansky-Aschoff sinus mimicking Rokitansky-Aschoff sinus with dysplasia ( $H$ and E, $\times 100$ ). (b) Diffuse carcinoembryonic antigen expression in the glands and along the lining of Rokitansky-Aschoff sinus in above case $(\times 400)$ (c) Mild-to-moderate dysplasia involving the lining of Rokitansky-Aschoff sinuses and gallbladder glands mimicking well-differentiated adenocarcinoma ( $H$ and $E, \times 100$ ). Carcinoembryonic antigen expressions were either negative or apical in the similar cases. ( $d$ and e) Adenomyomatosis of the gallbladder with mild-to-moderate dysplasia $(H$ and $E, \times 100)$ of glands with cytological and architectural atypia which have invaded into serosal layer. The RA sinus was not surrounded by desmoplastic stroma, rather the neoplastic glands invading the serosal layer were surrounded by dense desmoplastic stroma.

Therefore, we concluded that well-differentiated adenocarcinoma can mimic RA sinus. RA sinuses can be present focally or throughout the GB wall and some may extend into the muscular layer and perimuscular adipose tissues [Figure 2c]. RA sinuses have connection to the surface epithelium and are continuous structures having a smooth contour. However, adenocarcinoma usually comprises glands showing cytological atypia, irregular, and angulated contours and are arranged parallel to the surface. A prominent desmoplastic reaction usually favors adenocarcinoma over RA sinuses. Other associated features of adenocarcinoma glands are abnormal mitotic figures and intraglandular necrosis.

Giang et al. reported cases of pitfalls in diagnosis of $\mathrm{GBC}$, in which two cases were well-differentiated adenocarcinoma of GB mimicking RA sinuses. ${ }^{[14]}$

In one of the case, they reported small glands embedded in bundles of smooth muscle, surrounding a deep vessel but resembling RA sinus; however, the lining of deep glands presented cytological and subtle architectural atypia without significant inflammation, and the surrounding stroma was more desmoplastic than inflammatory in nature. Final diagnosis was well-differentiated adenocarcinoma with associated surface dysplasia. In another case, they found dysplasia extending into deeply situated RA sinuses, mimicking invasion. However, lateral intramural growth was also present at one place without typical associated stroma, in which atypical small glands were closely juxtaposed to smooth muscle. Final diagnosis was well-differentiated adenocarcinoma mimicking RA sinus with dysplasia.

Shirai differentiated RA sinus involvement by adenocarcinoma from stromal involvement by adenocarcinoma. ${ }^{[21]}$ The above study supported our observation that in case of dysplasia associated with RA sinus or when an adenocarcinoma progresses along RA sinus, it is not surrounded by a desmoplastic stroma. However, when malignant glands invade the stroma they are surrounded by intense desmoplastic stromal

Table 1: Chart showing different types of carcinoembryonic antigen expression and their percentages in benign gallbladder diseases with dysplasia and in gallbladder carcinoma cases

\begin{tabular}{|c|c|c|c|c|c|}
\hline CEA expression & $\begin{array}{l}\text { Number of benign GB diseases } \\
\text { with dysplasia (total cases - 39) }\end{array}$ & Percentage & CEA expression & $\begin{array}{c}\text { Number of GBC } \\
\text { cases - } 68\end{array}$ & Percentage \\
\hline No expression & $27 / 39$ & 69.2 & No expression & $6 / 68$ & 8.8 \\
\hline Apical & $12 / 39$ & 30.7 & Apical & $0 / 68$ & 0 \\
\hline Focal & $0 / 39$ & 0 & Focal & $29 / 68$ & 42.6 \\
\hline Diffuse & $0 / 39$ & 0 & Diffuse & $33 / 68$ & 48.5 \\
\hline
\end{tabular}

GB - Gallbladder; GBC - Gallbladder carcinoma; CEA - Carcinoembryonic antigen 
reaction. The author used the term "peri-RAS stroma" to denote the nondesmoplastic stroma surrounding the RA sinus associated with dysplasia or RA sinus involved by adenocarcinoma.

\section{Conclusion}

Some benign diseases of GB such as RA sinus and adenomyomatosis of GB may be misdiagnosed as GBC or vice versa. CEA immunomarker study may be helpful to resolve this diagnostic dilemma. In benign GB diseases, CEA expression is negative or apical; however, in majority of primary GBC, CEA expression is focal or diffuse.

\section{Financial support and sponsorship}

Nil.

\section{Conflicts of interest}

There are no conflicts of interest.

\section{References}

1. Misra S, Chaturvedi A, Misra NC, Sharma ID. Carcinoma of the gallbladder. Lancet Oncol 2003;4:167-76.

2. Diehl AK. Epidemiology of gallbladder cancer: A synthesis of recent data. J Natl Cancer Inst 1980;65:1209-14.

3. Dhir V, Mohandas KM. Epidemiology of digestive tract cancers in India IV. Gall bladder and pancreas. Indian J Gastroenterol 1999;18:24-8.

4. Kapoor VK, McMichael AJ. Gallbladder cancer: An 'Indian' disease. Natl Med J India 2003;16:209-13.

5. Randi G, Franceschi S, La Vecchia C. Gallbladder cancer worldwide: Geographical distribution and risk factors. Int J Cancer 2006;118:1591-602.

6. Bosman FT, Carneiro F, Hruban RH, Theise ND. WHO Classification of Tumours of the Digestive System. Vol. 4. Lyon: IARC Press; 2010. p. 266-74.

7. Roa I, Araya JC, Villaseca M, Roa J, de Aretxabala X, Ibacache G. Gallbladder cancer in a high risk area: Morphological features and spread patterns. Hepatogastroenterology 1999;46:1540-6.

8. Donohue JH, Nagorney DM, Grant CS, Tsushima K, Ilstrup DM, Adson MA. Carcinoma of the gallbladder. Does radical resection improve outcome? Arch Surg 1990;125:237-41.

9. Cubertafond P, Gainant A, Cucchiaro G. Surgical treatment of 724 carcinomas of the gallbladder. Results of the French Surgical
Association Survey. Ann Surg 1994;219:275-80.

10. Canil CM, Tannock IF. Doctor's dilemma: Incorporating tumor markers into clinical decision-making. Semin Oncol 2002;29:286-93.

11. Brandt-Rauf PW, Pincus M, Adelson S. Cancer of the gallbladder: A review of forty-three cases. Hum Pathol 1982;13:48-53.

12. Strom BL, Iliopoulos D, Atkinson B, Herlyn M, West SL, Maislin G, et al. Pathophysiology of tumor progression in human gallbladder: Flow cytometry, CEA, and CA 19-9 levels in bile and serum in different stages of gallbladder disease. J Natl Cancer Inst 1989;81:1575-80.

13. Kimura W, Shimada H, Kuroda A, Morioka Y. Carcinoma of the gallbladder and extrahepatic bile duct in autopsy cases of the aged, with special reference to its relationship to gallstones. Am J Gastroenterol 1989;84:386-90.

14. Giang TH, Ngoc TT, Hassell LA. Carcinoma involving the gallbladder: A retrospective review of 23 cases-Pitfalls in diagnosis of gallbladder carcinoma. Diagn Pathol 2012;7:10.

15. Agrawal V, Goel A, Krishnani N, Pandey R, Agrawal S, Kapoor VK. p53, carcinoembryonic antigen and carbohydrate antigen 19.9 expression in gall bladder cancer, precursor epithelial lesions and xanthogranulomatous cholecystitis. J Postgrad Med 2010;56:262-6.

16. Roa I, Araya JC, Wistuba I, De Aretxabala X, Salinas C, Mauriz A, et al. Immunohistochemical expression of the carcinoembryonic antigen in gallbladder cancer]. Rev Med Chil 1991;119:129-36.

17. Roa I, Araya JC, Shiraisch T, Yatani R, Wistuba I, Villaseca M, et al. Gallbladder cancer: Immunohistochemical expression of CA-19-9, epithelial membrane antigen, dupan-2 and carcinoembryonic antigen. Rev Med Chil 1992;120:1218-26.

18. Albores-Saavedra J, Nadji M, Morales AR, Henson DE. Carcinoembryonic antigen in normal, preneoplastic and neoplastic gallbladder epithelium. Cancer 1983 15;52:1069-72.

19. Van Patten K, Parkash V, Jain D. Cadherin expression in gastrointestinal tract endometriosis: Possible role in deep tissue invasion and development of malignancy. Mod Pathol 2010;23:38-44.

20. Albores-Saavedra J, Keenportz B, Bejarano PA, Alexander AA, Henson DE. Adenomyomatous hyperplasia of the gallbladder with perineural invasion: Revisited. Am J Surg Pathol 2007;31:1598-604.

21. Shirai Y. Histological differentiation of Rokitansky-Aschoff sinus involvement from stromal invasion of carcinoma of the gallbladder. Nihon Geka Gakkai Zasshi 1987;88:970-81. 